\title{
Direct Observation of the Flux-Line Vortex Glass Phase in a Type II Superconductor
}

\author{
U. Divakar, ${ }^{1}$ A. J. Drew, ${ }^{1}$ S. L. Lee, ${ }^{1}$ R. Gilardi,${ }^{2}$ J. Mesot, ${ }^{2}$ F. Y. Ogrin, ${ }^{3}$ D. Charalambous, ${ }^{4}$ E. M. Forgan, ${ }^{4}$ G. I. Menon, ${ }^{5}$ \\ N. Momono, ${ }^{6}$ M. Oda, ${ }^{6}$ C. D. Dewhurst, ${ }^{7}$ and C. Baines ${ }^{8}$ \\ ${ }^{1}$ School of Physics and Astronomy, University of St. Andrews, St. Andrews, Fife KY16 9SS, United Kingdom \\ ${ }^{2}$ Laboratory for Neutron Scattering, ETH Zürich and PSI Villigen, CH-5232 Villigen PSI, Switzerland \\ ${ }^{3}$ Department of Physics, University of Exeter, Exeter EX4 4QL, United Kingdom \\ ${ }^{4}$ School of Physics and Astronomy, University of Birmingham, Birmingham B15 2TT, United Kingdom \\ ${ }^{5}$ The Institute of Mathematical Sciences, C.I.T. Campus, Taramani, Chennai 600 113, India \\ ${ }^{6}$ Department of Physics, Hokkaido University, Sapporo 060-0810, Japan \\ ${ }^{7}$ Institut Laue-Langevin, 6, rue Jules Horowitz, B.P. 156-38042, Grenoble Cedex 9, France \\ ${ }^{8}$ Laboratory for Muon Spin Spectroscopy, PSI Villigen, CH-5232 Villigen PSI, Switzerland
}

(Received 29 June 2003; published 10 June 2004)

\begin{abstract}
The order of the vortex state in $\mathrm{La}_{1.9} \mathrm{Sr}_{0.1} \mathrm{CuO}_{4}$ is probed using muon-spin rotation and small-angle neutron scattering. A transition from a Bragg glass to a vortex glass is observed, where the latter is composed of disordered vortex lines. In the vicinity of the transition the microscopic behavior reflects a delicate interplay of thermally induced and pinning-induced disorder.
\end{abstract}

It has been acknowledged for many years [1] that the long range translational order of the Abrikosov vortex crystal in type II superconductors should be disrupted by arbitrarily small amounts of disorder. The current theoretical perspective is that weak disorder results in a "Bragg glass" (BG), which retains the topological order of the flux line lattice (FLL) but yields broadened diffraction peaks [2]. Strong disorder should result in the topologically disordered "vortex glass" (VG) [2]. The BG concept accounts for the observation of an apparently ordered FLL in many systems (e.g., [3,4]) and further evidence for this phase in an isotropic superconductor has recently been obtained using small-angle neutron scattering (SANS) [5]. At high magnetic fields and low temperatures, where the effects of disorder are stronger, the BG should be unstable to the formation of the VG. Here we report muon-spin rotation measurements on underdoped $\mathrm{La}_{1.9} \mathrm{Sr}_{0.1} \mathrm{CuO}_{4}$ (LSCO) which provide the first unambiguous experimental evidence for the transition from an ordered phase (BG) to a VG in a system of well-coupled vortex lines.

The importance of LSCO as a system in which to study the BG to VG transition can be understood in terms of two key parameters, namely, the superconducting penetration depth $\lambda_{a b}$ and the anisotropy parameter $\gamma$. The former determines the ability of the supercurrents in the copper-oxide sheets ( $a b$ planes) to screen externally applied magnetic fields, while the latter quantifies the uniaxial anisotropy of the penetration depth. In addition, the Josephson length $\lambda_{J}=\gamma s$ (where $s$ is the spacing of the copper-oxide planes), determines the effectiveness of currents, tunneling between the conduction planes, in maintaining the stiffness of a vortex line [6]. While higher values of $\lambda_{a b}, \gamma$ increase the susceptibility of the vortices to disordering, it is the ratio $\lambda_{J} / \lambda_{a b}$ which de- termines the dimensionality of the vortex system. For example, in optimally doped $\mathrm{YBa}_{2} \mathrm{Cu}_{3} \mathrm{O}_{7-\delta}$ (YBCO) $\lambda_{J} / \lambda_{a b} \ll 1$, so the vortices resemble the rigid rods of flux found in conventional isotropic superconductors. In contrast, for optimally doped $\mathrm{Bi}_{2} \mathrm{Sr}_{2} \mathrm{CaCu}_{2} \mathrm{O}_{8+\delta}$ (BSCCO) $\lambda_{J} / \lambda_{a b} \gtrsim 1$; consequently the vortex system is quasi-two dimensional. As we demonstrate here, the novelty of underdoped LSCO is that the material parameters $\left(\lambda_{a b} \sim 3000\right.$ and $\left.\lambda_{J} \sim 250 \AA\right)$ give rise to a system of fairly rigid vortex lines which are nonetheless highly susceptible to transverse fluctuations.

Our muon spin rotation ( $\mu \mathrm{SR})$ measurements were performed using the GPS spectrometer on the $\pi \mathrm{M} 3$ beam line at the Paul Scherrer Institut, Switzerland. The field was aligned to within $5^{\circ}$ of the $c$ axis of the crystal, and the muon spins were initially polarized nearly perpendicular to the applied field. The muons implant in the sample at random positions with respect to the vortices, and each precesses at a rate $\omega=\gamma_{\mu} B(r)$, determined by the local field $B(r)$ and the gyromagnetic ratio of the muon. The muon-spin precession is monitored via the positrons which are emitted preferentially along the muon-spin direction during the decay of the muon [7]. The precession signal, averaged over $\sim 10^{7}$ muons, reveals the probability distribution $p(B)$ of the spatial variation $B(r)$. The sample was a single crystal of length $20 \mathrm{~mm}$ and diameter $5 \mathrm{~mm}$, with the $c$ axis perpendicular to the axis of the cylinder. The superconducting transition temperature of the sample was $T_{c}=29 \mathrm{~K}$ with a width $(10 \%-90 \%) \Delta T=1.5 \mathrm{~K}$, indicating the high quality of the sample. All $\mu$ SR measurements were made after cooling in an applied field.

In a $\mu$ SR experiment the presence of a vortex line lattice is revealed by a $p(B)$ which has a distinct signature, most notably a peak followed by a tail extending 
towards fields higher than the mean, reflecting the small volume of the core region of the vortices. The peak at the mode of the distribution represents the high probability density of the field value at the center of a line connecting two vortex cores [7]. In Fig. 1(a) are shown probability distributions measured in LSCO at low field, where those taken at the lowest temperatures correspond most closely to the line shape expected from an ideal lattice. One of the calculated line shapes given in Fig. 1(c) is also derived from an ideal vortex lattice, where for clarity the rounding effects of instrumental resolution and nuclear dipolar broadening, visible in the experimental data of Fig. 1(a), have been omitted. The muon probe measures the local field, which is determined by contributions from vortices only within $\sim \lambda_{a b}$ of the muon; hence, the general form of $p(B)$ is remarkably robust to changes in long range order [8]. In contrast, the technique can be highly sensitive to changes in the local environment due to thermally induced or pinning-induced distortions [7], which allows us to observe the transition from the ordered state to the VG phase in LSCO.

The main result of this Letter can be appreciated immediately by comparing the normalized experimental line shapes of Figs. 1(a) and 1(b), measured at 80 and $6 \mathrm{kOe}$, respectively. Referring first to the lowest temperature line shape at each field, it is clear that they are very different. While the 80 Oe distribution has the characteristics of an ideal vortex lattice described above, the $6 \mathrm{kOe}$ signal is highly symmetric, indicating a strong departure from an ideal vortex lattice or Bragg-glass arrangement. For comparison, in Fig. 1(c) we include the $p(B)$ derived from Monte Carlo simulations of a perfect triangular Abrikosov lattice and also for a vortex glass structure having short-ranged translational correlations of the order of 6 intervortex spacings. Within this scenario, discussed in Ref. [9], the system undergoes a transition from a $\mathrm{BG}$ phase to a multidomain glass comprising a size distribution of domains within which the vortices are locally ordered. Increasing the field leads to a rapid fall in the average domain size just above the BG phase. Note that the distribution function obtained for the multidomain structure is far more symmetric as well as broader than is the case for the perfect lattice, as is found experimentally in the data at $6 \mathrm{kOe}$ [see Fig. 1(b)].

Further conclusive evidence for the transition from a BG to a VG phase comes from examining the width of the line shapes $\sigma$, given by the square root of the second moment of $p(B)$. This quantity is plotted in Fig. 2 as a function of applied field, for a range of temperatures. For data taken at the lowest temperatures, it can be seen that the signal measured at $6 \mathrm{kOe}$ is considerably broader than that at 80 Oe. It has been shown theoretically $[10,11]$ that such a broadening of the signal from the vortex lattice can only arise from static disorder in the positions of vortex lines within a plane perpendicular to the field. This arises due to positional disorder of the vortices, which gives rise to regions with field values both higher and lower than in the well-ordered lattice [8,10-12]. Conversely, in a system composed of two-dimensional "pancake-vortex" strings [10], short-wavelength transverse fluctuations along the field direction would always lead to a narrowing of the field distribution $p(B)$ [10], as observed experimentally in the very anisotropic (quasitwo-dimensional) BSCCO [13]. Thus it is clear that in LSCO we are dealing with a highly disordered vortex line arrangement, which we identify with the VG phase.

We now turn our attention to the temperature dependence of the line shapes of Fig. 1(a), where it can be seen that increasing temperature truncates the high-field tails. This reflects the increasing amplitude of thermally induced fluctuations $\left\langle u^{2}(T)\right\rangle^{1 / 2}$ of the vortex positions, on a time scale faster than the characteristic muon sampling time [14]. The muons thus experience a time-averaged field distribution in which the high-field values arising from close to the vortex cores are smeared out. Compared to those at low temperature, the line shapes are narrowed. Such narrowing arises both from the increase in $\lambda(T)$ and $\left\langle u^{2}(T)\right\rangle^{1 / 2}$. This may be described by $[14,15]$ :
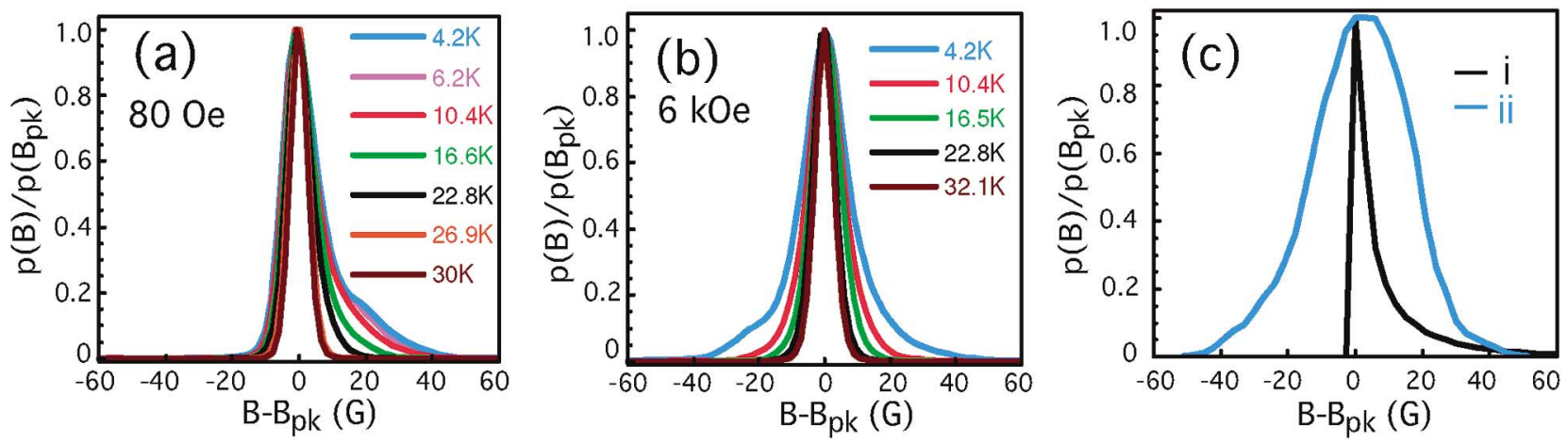

FIG. 1 (color). (a) Temperature-dependent probability distributions of the internal field, $p(B)$, due to the presence of the flux lines at applied fields of (a) 80 Oe and (b) $6 \mathrm{kOe}$. (c) Field distributions derived from Monte Carlo simulations of the vortex state at 6 kOe (see text) for the case of: (i) an ideal vortex lattice ; (ii) a VG phase with a transverse correlation length of about six lattice spacings. The latter also includes a small contribution due to the effects of thermal narrowing (as observed experimentally). All curves in the figure are normalized at the mode of the distribution to allow easy comparison of the shapes. 


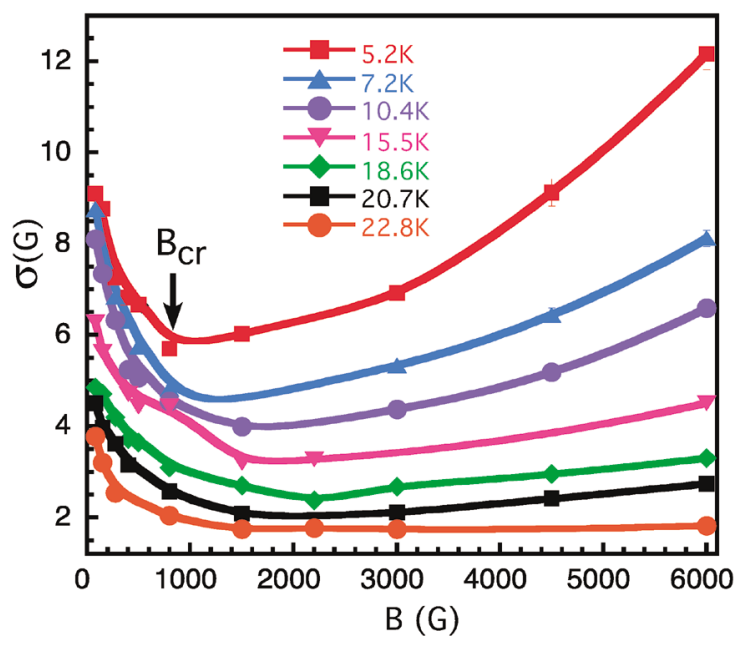

FIG. 2 (color). (a) The variation with field of the linewidth $\sigma$ at selected temperatures. The initial reduction with increasing field corresponds to the field dependent influence on the field distribution of both thermal fluctuations and increasing elastic vortex interactions. Above $B_{\mathrm{cr}} \sim 1 \mathrm{kOe}$, the width dramatically rises due to the onset of static disorder in the VG phase. Note that the high-field dependence has almost disappeared by a temperature of $23 \mathrm{~K}$, and disappears completely at the irreversibility line (see Fig. 4).

$$
\sigma^{2}(T)=\langle B\rangle^{2} \sum_{\vec{\tau} \neq 0} \frac{e^{-\tau^{2}\left\langle u^{2}\right\rangle / 2}}{\left[1+\lambda^{2}(T) \tau^{2}\right]^{2}},
$$

where the $\vec{\tau}$ are reciprocal lattice vectors, multiples of $\pi / d$, and $d \sim \sqrt{\Phi_{o} / B}$ is the plane spacing of the vortex lattice. At a given temperature, $\sigma$ decreases as the field is increased, mainly due to an increase in the ratio $\left\langle u^{2}\right\rangle^{1 / 2} / d$. This is in contrast to the case of an ideal static vortex lattice, where $\sigma$ is independent of field in the range $H_{c_{1}}<H \ll H_{c_{2}}$. In Fig. 2 one observes a reduction in $\sigma$ with increasing field up to fields of around $1 \mathrm{kOe}$. While the observed changes of the line shapes show that this must at least partly be due to these dynamical effects, simulations indicate that the magnitude of this narrowing cannot be entirely attributed to this mechanism. The broadening towards lower fields must also include a contribution from an increase in the static disorder due to the reduction of elastic interactions between vortices in this very dilute vortex state. Indeed, it is only at fields above a few hundred Oersted that the vortex separation becomes less than the penetration depth in this system, whereby the shear modulus for the lattice increases significantly [6]. A minimum in $\sigma(B)$ thus occurs due to the competition of these broadening mechanisms in the vicinity of the transition to the VG phase.

We have also recently used SANS to perform a preliminary experiment to image the vortex arrangement in the same LSCO sample, using instrument D22 at the Institut Laue-Langevin, Grenoble, France. At an applied field of 150 Oe a diffraction pattern could be measured [Fig. 3(a)], thus confirming the quasi-long-range order of the vortex lattice at low fields. At a temperature of $5 \mathrm{~K}$ the intensity was found to drop very rapidly as a function of applied field. Furthermore, the diffraction spots show an appreciable broadening in $q$ in the vicinity of $B_{\mathrm{cr}} \sim$ $800 \mathrm{G}$, which, in accord with the $\mu \mathrm{SR}$ data, implies an increase of static disorder in the system around this field. While in the $\mu$ SR experiment a field-cooled (FC) experiment was necessary to interpret the line shapes, in the SANS experiment we attempted to improve the lattice perfection in the Bragg-glass region by cooling in a modulated applied field, as in Ref. [16]. The lattice perfection was always found to be reduced by these approaches, indicating that the FC state for both SANS and $\mu \mathrm{SR}$ represents the most ordered vortex state at low fields.
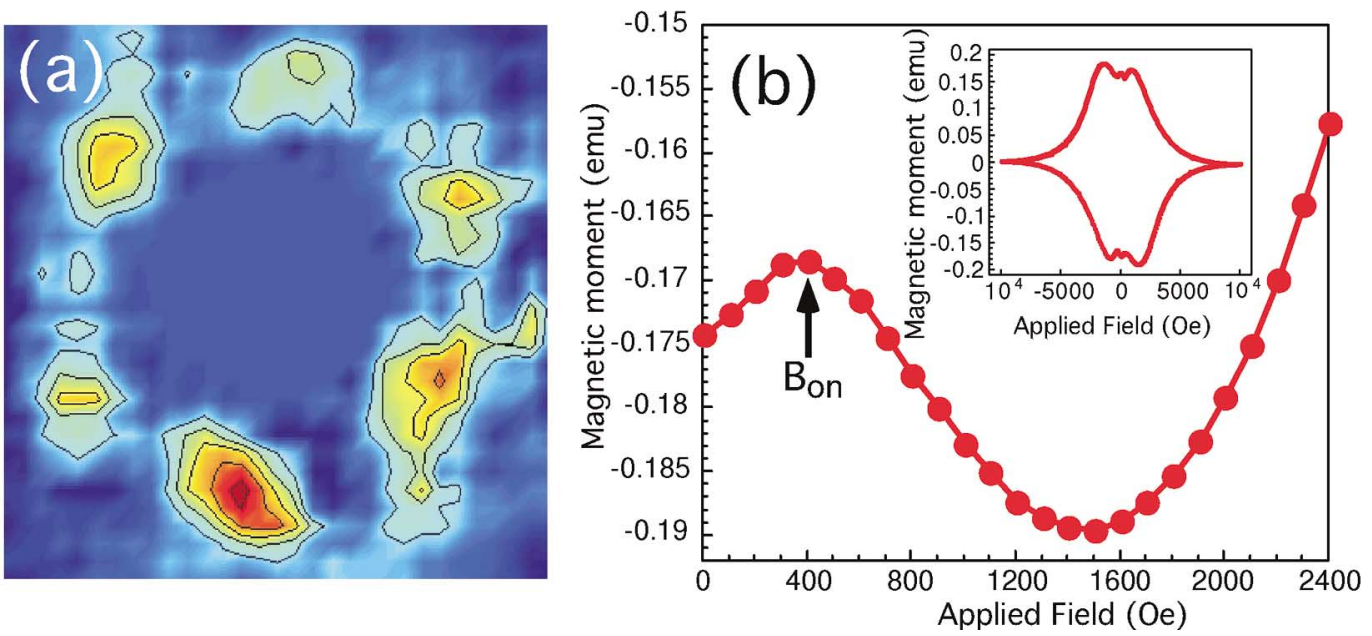

FIG. 3 (color). (a) A small angle neutron diffraction pattern showing the existence of an ordered vortex phase at low fields (150 Oe) and temperature $(6.2 \mathrm{~K})$. The intensity of this pattern rapidly falls as the field is increased towards the VG phase (see text). (b) The feature in the magnetization at $16.5 \mathrm{~K}$ is associated with the crossover to the VG state, measured on a small piece taken from the $\mu \mathrm{SR}$ sample. The inset shows a complete hysteresis loop. 


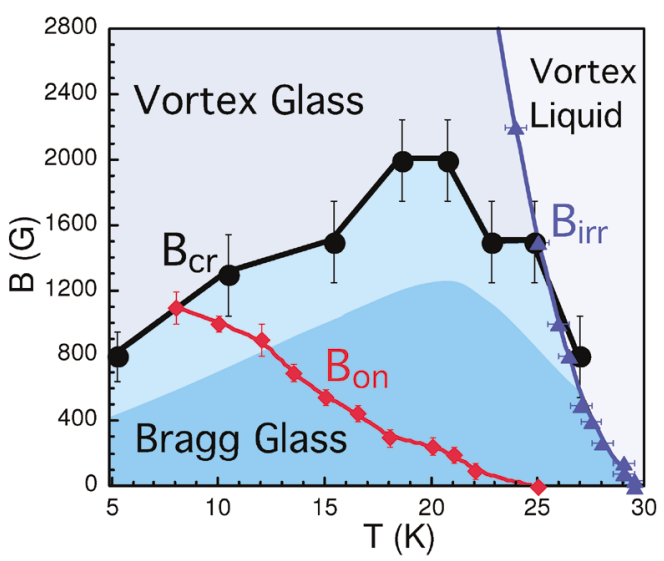

FIG. 4 (color). The magnetic phase diagram derived from the changes observed in the $\mu$ SR field distributions. $B_{\mathrm{cr}}(T)$ indicates the onset of the broadening at high field, which is significant only below $\sim 25 \mathrm{~K}$, and should be considered as an upper limit for the BG-VG transition (see text). This uncertainty in the exact position of the transition is represented schematically by the shading below the line $B_{\mathrm{cr}}(T) . B_{\text {irr }}(T)$ indicates the irreversibility line as determined by bulk measurements of the field-cooled-zero-field-cooled (FC-ZFC) magnetisation using a SQUID magnetometer. The position of the feature in the magnetisation curves $B_{\text {on }}(T)$ is also plotted [see Fig. 3(b)].

In the literature, evidence for the existence of the BGVG transition is frequently taken from signatures in the bulk magnetization, since the transition to the glassy state is accompanied by an increased effectiveness of point pinning. In Fig. 3(b) is a section of an $M(H)$ loop at $16.5 \mathrm{~K}$, where a "peak effect" is evident as an anomalous increase in the magnitude of the magnetization beginning at around 400 Oe. The temperature dependence of the position of the onset of this peak $B_{\text {on }}(T)$ is plotted in the phase diagram of Fig. 4. Similar features have previously been associated with the BG-VG transition, e.g., [17].

The occurrence of an equilibrium phase transition with increasing magnetic field has been discussed theoretically by many authors, e.g., [2]. It is worth noting that the muon is sensitive only to the local magnetic field, so in general this transition will be manifest as a crossover of behavior which reflects the underlying sharp transition. In Fig. 4 the line $B_{\mathrm{cr}}(T)$ is a plot of the minima in $\sigma(B)$, where the broadening due to static glassy disorder begins to dominate. This feature thus provides an upper limit for the BGVG transition. An upward trend of this BG-VG transition, reminiscent of that observed experimentally, $B_{\mathrm{cr}}(T)$, has been predicted to arise from the reduced effectiveness of pinning at higher temperature $[2,18]$. The dependence of $B_{\text {on }}(T)$ is very different to $B_{\mathrm{cr}}(T)$. This is not surprising given that the muons measure $p(B)$ in a FC state, whereas the magnetization measurements determine the macroscopic properties of a disturbed system possessing strong macroscopic flux gradients, and reflect the changing dynamic response to the pinning landscape.

In conclusion, we have measured the vortex state at a microscopic level and observed a change with field from a $\mathrm{BG}$ to a VG phase, the latter possessing only short range transverse order. The minimum in the $\mu$ SR linewidth with increasing field, observed at $B_{\mathrm{cr}}(T)$, arises primarily from the competing influences of increasing elastic interactions combined with thermal fluctuations, and a rapid collapse of translational correlation lengths in the vortex system within the plane perpendicular to the field direction. This provides unambiguous evidence for a transition with increasing field from a nominally Bragg-glass phase to a more disordered vortex glass state. This is the first such measurement on a system composed of well-coupled vortex lines, and paves the way for a detailed study of the evolution of short range order in the presence of weak pinning, a problem of universal significance [2].

This work was supported by the Swiss National Science Foundation, the Engineering and Physical Sciences Research Council of the United Kingdom, the DST (India) and the Ministry of Education, Science and Technology of Japan. The $\mu$ SR experiments were performed at the Swiss Muon Source, Paul Scherrer Institute, Villigen.

[1] A. I. Larkin and Y.V. Ovchinnikov, J. Low Temp. Phys. 34, 409 (1979).

[2] T. Giamarchi and P. Le Doussal, Phys. Rev. Lett. 72, 1530 (1994).

[3] R. Cubitt et al., Nature (London) 365, 407-411 (1993).

[4] R. Gilardi et al., Phys. Rev. Lett. 88, 217003 (2002).

[5] T. Klein et al., Nature (London) 413, 404 (2001).

[6] G. Blatter et al., Phys. Rev. B 54, 72 (1996).

[7] C. M. Aegerter and S. L. Lee, Appl. Magn. Res. 13, 75 (1997).

[8] E. H. Brandt, J. Low Temp. Phys. 73, 355 (1988).

[9] G. I. Menon, Phys. Rev. B 65, 104527 (2002).

[10] E. H. Brandt, Phys. Rev. Lett. 66, 3213 (1991).

[11] G. I. Menon et al., Phys. Rev. B 60, 7607 (1999).

[12] E. H. Brandt, Phys. Rev. B 37, 2349 (1988).

[13] S. L. Lee et al., Phys. Rev. Lett. 71, 3862 (1993).

[14] Y.-Q. Song et al., Phys. Rev. Lett. 70, 3127 (1993).

[15] S. L. Lee et al., Phys. Rev. Lett. 75, 922 (1995).

[16] S. J. Levett, C. D. Dewhurst, and D. McK. Paul, Phys. Rev. B 66, 014515 (2002).

[17] D. Giller et al., Phys. Rev. Lett. 79, 2542 (1997).

[18] Denis Ertas and David R. Nelson, Physica (Amsterdam) 272C, 79 (1996). 